\title{
La question du passif en classe de FLE en Tunisie vue à travers l'observation d'une séance de grammaire
}

\author{
The question of the passive voice in FLE class in Tunisia seen \\ through a grammar session observation
}

\section{Mohamed Ben Ammar}

Université de Gafsa, Tunisie

\begin{abstract}
Résumé : La grammaire telle qu'elle se présente dans les manuels scolaires de FLE subit une certaine transformation au moment de son enseignement en classe sous l'influence notamment de la méthode adoptée par l'enseignant et des choix terminologiques que celui-ci endosse. À travers l'observation d'une séance de grammaire portant sur la question du passif en classe de FLE en Tunisie, cet article tente de mettre en lumière certaines pratiques enseignantes ainsi que les défaillances dans la culture grammaticale des élèves. L'écart entre la grammaire destinée à être enseignée et celle enseignée effectivement en classe traduit l'importance des pratiques grammaticales, et témoigne du rôle capital de celles-ci dans le développement de la compétence métalinguistique des apprenants.
\end{abstract}

Mots-clés : grammaire, classe de FLE, le passif, pratiques enseignantes.

Abstract: Grammar as it appears in FLE (French as a foreign language) textbooks will undergo a certain transformation at the time of its teaching in the classroom under the influence in particular of the method adopted by the teacher and the terminological choices that this one endorses. Through the observation of a grammar session about the issue of the passive (voice) in a FLE classroom in Tunisia, this article attempts to highlight some teaching practices as well as failures in the grammatical culture of students. The gap between the grammar intended to be taught and that actually taught in classroom reflects the importance of grammatical practices and proves to their crucial role in the development of the learners' grammatical competence.

Keywords: grammar, FLE (French as a foreign language) classroom, the passive (voice), teaching practices.

\section{Introduction}

Le présent article aborde le problème de la structuration interne de l'enseignement grammatical au collège du point de vue des pratiques de classe. Cette étude empirique observe de près la classe de français au cours des activités grammaticales, c'est-à-dire qu'elle essaie d'éclairer certains aspects de l'enseignement de la grammaire

conçu comme le processus interactif qui a cours dans la classe de langue lorsqu'il y a présentation d'un point de grammaire ou échange ou discussion sur quelque aspect grammatical de la langue cible (Germain, Séguin, $1998: 155)$. 
La grammaire des manuels scolaires subira un certain nombre de transformations lorsqu'elle sera exposée aux élèves en situation d'enseignement-apprentissage. L'intérêt de l'observation de la grammaire en classe ou ce qu'on appelle "grammaire interactive » réside dans le fait que celle-ci participe en grande partie à la construction de la compétence grammaticale de l'apprenant. De telles observations permettent également de mesurer l'écart entre les pratiques des enseignants en situation d'enseignement-apprentissage et les directives et recommandations proposées par le cadre officiel. On sait que les méthodes et les techniques d'enseignement sont multiples, diverses et que souvent elles se chevauchent. La situation est telle, l'enseignant se trouve dans l'obligation de sélectionner celles qui paraissent les mieux adaptées à la situation de sa classe.

Quant à la tradition didactique, elle préconise un processus classique de l'enseignement grammatical fondé sur l'explication des règles par le professeur ef l'exécution des exercices par les élèves. Or incontestablement, l'enseignant a besoin de suivre le développement du savoir et donc de renouveler ses pratiques afin qu'elles soient en adéquation avec les nouvelles orientations des grammairiens et des linguistes. C'est ainsi que ces pratiques pourraient s'adapter aux besoins langagiers des apprenants.

Par ailleurs, depuis un certain temps, la réflexion didactique tend à favoriser la perspective actionnelle en classe de langue : les élèves jouent un rôle central parce qu'ils exécutent la quasi-totalité des activités, c'est-à-dire qu'ils découvrent des règles de grammaire et inter-corrigent les productions. Cette perspective est étroitement liée à la notion de tâche que le CECRL définit comme :

\section{[...] toute visée actionnelle que l'auteur se présente comme devant parvenir à un résultat donné en fonction d'un problème à résoudre, d'une obligation à remplir, d'un but qu'on s'est fixé (Conseil de l'Europe, $2001: 16)$.}

Dans cette recherche, nous allons rapporter l'essentiel de l'observation d'une séance portant sur une question majeure de la grammaire française (la transformation passive), qui figure dans le manuel de ge année. Nous insisterons sur la démarche adoptée par l'enseignant, puis sur l'interaction verbale en classe qui va nous révéler quelques difficultés grammaticales rencontrées par les élèves ainsi que les techniques d'enseignement et le discours grammatical du professeur.

\section{Présentation du corpus}

Dans ce travail, nous avons eu recours à une méthode de recherche reconnue en éducation, l'étude de cas. Nous avons en effet observé les pratiques d'un enseignant de français langue étrangère dans la classe de 9e année de base (Classe terminale du collège en Tunisie avec des élèves de 15 à 16 ans) relativement à quelques dimensions spécifiques de l'enseignement-apprentissage d'une question de la grammaire phrastique: le passif. L'analyse du contenu de la séance enregistrée part de la comparaison des contenus grammaticaux du manuel avec les connaissances dispensées effectivement en classe de FLE. C'est là l'occasion de mesurer l'écart entre la grammaire d'enseignement et l'enseignement de la grammaire. Bien entendu, l'intervention de l'enseignant est à l'origine de ce décalage dans la mesure où sa propre expérience et la conception qu'il se fait de l'activité grammaticale dessinent, en grande partie, les contours de son action 
didactique. L'élève subit les effets d'une telle différence entre les deux types de grammaire et se trouve généralement tiraillé entre le manuel et l'enseignant.

\subsection{Présentation de la séance}

Thème : La transformation passive.

Niveau : 9e année de l'enseignement de base.

Date d'enregistrement : 15/02/2010.

Lieu d'enregistrement : Ecole Préparatoire ENNAJAH- Redeyef .Gafsa

Support de la séance : corpus phrastique.

$\mathrm{Pl}$ : Des ingénieurs japonais ont construit le stade.

P2 : Les élèves feront l'exercice.

P3 : On a battu l'équipe de flage.

P4 : Robert parle à sa mère.

- Objectifs de la séance : amener les élèves à la maîtrise de la transformation passive.

- Emplacement de la séance par rapport à l'ensemble du programme : cette séance appartient au module d'apprentissage $n^{\circ} 3$, elle intervient alors que les élèves ont déjà étudié les questions grammaticales suivantes: I'expression du temps, les expansions du groupe nominal, les verbes d'état, les procédés de reprise, les pronoms personnels C.O.D et C.O.I.

\subsection{Remarques préliminaires}

Il faut noter tout d'abord que l'activité grammaticale au collège revêt la forme d'une séance isolée fondée sur l'explication ou la découverte des règles et l'utilisation d'un discours métalinguistique adéquat, voire adapté à la situation d'enseignement-apprentissage.

- Par rapport au titre proposé par le manuel (la forme passive), le choix du professeur (la transformation passive) paraît plus adéquat aux objectifs de la séance et au savoir présenté par le manuel lui-même.

- Contrairement au manuel qui construit la phase d'explication autour d'un texte, l'enseignant opte pour un corpus phrastique, lequel représente à ses yeux un support idéal pour l'étude du passif car il lui permet de choisir des énoncés illustrant les idées qu'il veut intégrer dans son explication. Cependant, les textes officiels préconisent, conformément aux avancées de la recherche en didactique des langues, de mettre les points grammaticaux enseignés au service de la compétence communicative. Ce qui veut dire que l'enseignement de la grammaire à partir de phrases isolées et coupées de leur contexte contredit, d'une part, les principes didactiques imposés par les textes officiels; et entrave le développement d'une compétence communicative chez les apprenants, d'autre part. Ainsi, pour atteindre l'objectif fixé par l'institution, l'enseignant doit choisir le corpus le moins artificiel possible afin de se rapprocher de plus en plus des situations authentiques de la production langagière. Dans ce sens, Vigner affirme qu': "il faut faire en sorte que la classe soit le lieu d'acquisition d'un savoir grammatical par lequel, s'il est bien 
conçu, l'apprenant disposera de cette faculté à combiner en suites acceptables des unités de langue. Sans quoi toute volonté de communiquer se heurte immanquablement aux ambigüités, imprécisions, malentendus auxquels peut donner lieu l'usage d'un code mal maitrisé ॥ (2004 : 107).

- Le support de la séance est formé de quatre phrases qui traduisent le raisonnement du professeur s'agissant de l'organisation de l'information. Les deux premières phrases admettent la transformation passive et serviront donc d'exemples pour expliquer aux élèves le mécanisme de passivation. La troisième phrase a pour sujet le pronom personnel indéfini on, ce qui permettra d'étudier le phénomène de l'effacement du complément d'agent; la quatrième contient un complément d'objet indirect (C.O.I) et illustrera l'impossibilité de la transformation passive.

Ainsi, nous pouvons dire que le professeur adopte une stratégie classique, qui se construit autour d'une explication du point grammatical en question, et qui sera suivie, dans un deuxième temps, d'une étude des cas particuliers ou des exceptions.

\subsection{La démarche}

Il est primordial que la pratique de la grammaire en classe de $\mathrm{L}_{2}$ soit adéquate aux intentions des programmes officiels. Ceux-ci requièrent l'intégration de la grammaire aux activités lecturales et scripturales en vue de l'amélioration de la compétence communicative des apprenants.

Dans cette optique, les dernières avancées de la recherche en didactique de la grammaire préconisent le recours à la grammaire sémantique qui incarne l'esprit de l'approche communicative en privilégiant le sens au détriment de la forme. Dans les pratiques de classe, il existe deux types de démarche : soit la démarche sémasiologique qui part de la forme pour atteindre le sens, soit la démarche onomasiologique qui part du sens pour arriver à la forme. Cette dernière tendance traduit le penchant naturel de tout apprenant de langue étrangère à communiquer du sens à l'aide des formes qui lui correspondent.

L'examen de la séance que nous avons observée montre que l'enseignant a eu recours à deux approches différentes voire opposées, lesquelles correspondent aux deux grandes phases de la leçon qu'il a présentée.

La première phase consiste à identifier la forme active des phrases constituant le corpus en s'appuyant sur une définition sémantique, comme il apparaît dans la séquence suivante (nous n'avons pas rapporté les blocages des élèves qui seront analysés ultérieurement) :

$P$ : Qui fait l'action dans ces phrases ? Dans la première phrase ?

$\mathrm{E}:$ «Des ingénieurs ».

$P$ : Quelle est la fonction « Des ingénieurs»?

E : Sujet.

$P$ : Qui fait l'action dans toutes ces phrases?

E : C'est le sujet qui fait l'action.

$\mathrm{P}$ : Vous avez dit que le sujet fait l'action dans chacune de ces phrases 
Comment appelle-t-on une phrase dont le sujet fait l'action?

(Le professeur n'a pas reçu de réponse, il a posé la question autrement)

$P$ : S'agit-il de phrases actives ou passives?

E : Lorsque le sujet fait l'action, il s'agit d'une phrase active.

$P$ : Ce sont donc des phrases actives parce que tous les sujets sont actifs.

$P$ : Quand est-ce qu'un sujet est dit actif ?

E : Quand il fait l'action.

À partir des structures précédentes, l'enseignant adopte une stratégie pour mener à la conclusion que le corpus est formé de phrases actives dont le sujet fait l'action. Au cours de cette phase, le groupe-classe est en situation sémasiologique. Une pareille stratégie s'explique vraisemblablement par le souci de l'enseignant de préparer la phase la plus importante de la leçon, à savoir celle de la transformation passive qui se fonde sur une définition sémantique de la phrase passive. La première phase constitue donc un socle à partir duquel l'enseignant va énoncer une définition de la forme passive pour retrouver la structure syntaxique qui lui correspond, comme le prouve l'extrait suivant :

$P$ :On passe maintenant au mécanisme de la transformation passive. Contrairement à la phrase active, dans la phrase passive, au lieu de faire l'action, le sujet...?

E : Le sujet subit l'action.

P : Que devient le C.O.D de la phrase active?

$\mathrm{E}:$ Il devient sujet.

$\mathrm{P}:$ Et, le sujet de la phrase active, que devient-il ?

Absence de réponse.

P:Le sujet de la phrase active devient complément d'agent de la phrase passive.

$P$ : En ce qui concerne le verbe, il garde la même place, il est formé de l'auxiliaire "être " conjugué au même temps que le verbe de la phrase active plus le participe passé du verbe.

Après avoir explicité le processus de passivation, le professeur passe à la transformation des phrases du corpus.

La seconde approche, davantage prônée par les tenants de la conception communicative de l'enseignement des langues, repose sur le passage du sens à la forme linguistique. En effet, l'enseignant a commencé par une définition de la phrase passive en tant que phrase dont le sujet subit l'action pour amener les élèves à remarquer que le premier changement, dans le processus de passivation, consiste à mettre le C.O.D de la phrase active en position de sujet dans la phrase passive.

À ce moment-là, le groupe-classe est en situation onomasiologique. À ce propos, J. Courtillon (1989) conseille de recourir à l'approche onomasiologique dès les premiers moments de l'apprentissage pour empêcher l'élève d'assimiler, par 
analogie, les valeurs d'une forme grammaticale de la langue étrangère à une autre forme de sa langue maternelle.

Cet enseignant, semble-t-il, met en œuvre plus d'une approche grammaticale, il varie sa démarche selon la situation dans laquelle il se trouve; l'essentiel pour lui est de communiquer des explications grammaticales fiables et surtout accessibles à la majorité des élèves.

Cependant, nous ne pouvons pas nous empêcher de souligner l'existence d'un certain éclectisme dans l'enseignement d'une question relevant de la grammaire de la phrase. Un tel procédé n'est pas justifié car on ne peut prouver son efficacité de manière définitive, comme le note Cicurel $(1985: 65)$ :

On pourra ici osciller entre deux interprétations de ce comportement : l'éclectisme est-il synonyme de confusion, car l'apprenant s'embrouille dans les explications successives ou, au contraire, cette multiplicité des procédés explicatifs augmente-t-elle les chances des apprenants de comprendre l'énoncé ?

Néanmoins, il n'est pas un seul enseignement de la grammaire qui ne recourt à la multiplication ou à la variation des techniques. Dans cette perspective, J-C. Beacco (1995: 45) observe que le recours à l'éclectisme dans les pratiques de la classe de langue se fait essentiellement par une sélection raisonnée et non par un rassemblement hétéroclite de techniques d'enseignement. En d'autres termes, en garantissant la cohérence des techniques empruntées, on évite toute sorte de "bricolages " risquant d'empêcher l'apprenant de participer activement au processus d'enseignement-apprentissage. Selon ce chercheur, l'éclectisme est généralement perçu comme une "méthodologie circulante " sur laquelle les méthodologies constituées viennent s'empiler. Ce choix évite une démarche dogmatique et unidimensionnelle, et permet de subvenir aux besoins de l'apprenant. Le choix éclectique s'impose car il n'y a pas une méthode qui puisse à elle seule répondre à la diversité des besoins d'une classe hétérogène.

\section{Résultats et discussion}

\subsection{L'interaction}

La classe de langue est un véritable laboratoire d'échanges verbaux; ces derniers couvrent en effet l'ensemble des discours produits par l'enseignant et/ou les élèves.

La communication au sein de cet espace éducatif est une action pédagogique entreprise pour réaliser les objectifs d'une leçon, d'un module d'apprentissage ou d'un programme d'enseignement. L'interaction entre les deux partenaires du triangle didactique vise à la construction d'un savoir et à l'apprentissage voire à la compréhension des connaissances qui seront par la suite mises en application afin d'être intériorisées une fois pour toutes. C'est là un moment crucial du processus d'enseignement-apprentissage.

En matière de grammaire, nous avons affaire à des notions qui ont évolué d'une époque à l'autre, d'une théorie à l'autre, d'un modèle de référence à l'autre. De ce fait, il est fort probable que les élèves aient fréquenté des modèles autres que ceux auxquels les enseignants font référence. Dans ce cas, on peut assister à des situations conflictuelles dues à «l'hétérogénéité du répertoire grammatical des 
apprenants et de l'enseignant ॥ (Cuq, 1996:72), ce qui peut gêner l'interaction et empêcher l'intercompréhension des acteurs de la classe.

De façon plus générale, l'activité grammaticale en classe de $\mathrm{L}_{2}$ pose un bon nombre de problèmes, aussi bien aux enseignants qu'aux enseignés: il suffit, par exemple, que l'enseignant fasse usage d'une métalangue non connue des apprenants, pour que l'on se retrouve dans une situation où le processus communicationnel risque de s'interrompre. Nous essayerons, à travers ces portions de communication, de mettre en évidence quelques problèmes grammaticaux des élèves et d'éclairer, en même temps, certaines pratiques des enseignants.

\subsubsection{Les problèmes rencontrés par les élèves}

L'arrivée soudaine d'une nouvelle génération d'élèves au collège, de niveaux plus hétérogènes, la mise en place d'une nouvelle approche didactique des questions morphosyntaxiques, la diversification des manuels du second cycle de l'école de base influent actuellement sur la connaissance grammaticale des apprenants.

De fait, une analyse des séances de grammaire montre que les difficultés rencontrées se rapportent essentiellement à une insuffisance de la culture grammaticale, phénomène qui ne se manifestait pas de façon aussi nette chez les générations précédentes. Une leçon traitant la question de la transformation passive fait apparaître des lacunes dont nous exposerons les plus révélatrices.

\subsubsection{Méconnaissance du pronom personnel indéfini On}

L'une des articulations de la leçon porte sur l'effacement de l'agent de l'action dans la phrase passive. L'enseignant a choisi le cas de la phrase active qui a pour sujet le pronom personnel indéfini on. La reconnaissance de ce pronom n'est pas une tâche aisée pour l'ensemble de la classe. La portion de communication suivante en est la preuve :

$P$ : Quel est le sujet de la phrase 3 ?

E : C'est On.

$P$ : Quelle est la nature grammaticale de On?

E : Absence de réponse.

$P$ : Que désigne On? Une personne ou plusieurs personnes?

E : Une personne.

$\mathrm{P}$ : Comment vous le savez?

$E$ : il se conjugue au singulier.

$P$ : Est-ce qu'on connaît précisément cette personne? Est-ce qu'on peut remplacer on par un groupe nominal ?

E : Non.

$P$ : Donc, il s'agit d'un pronom personnel inconnu, nous ne pouvons pas l'identifier, il peut désigner une ou plusieurs personnes, c'est un pronom personnel indéfini.

Cette séquence met en évidence une difficulté grammaticale liée à l'identification du pronom personnel indéfini on. Cette insuffisance dans la culture 
grammaticale des élèves tient essentiellement au fait que les enseignants négligent ce pronom qui ne figure pas dans les exercices de conjugaison réalisés en classe.

En l'absence d'informations sur ce point, les élèves partent du principe qu'un pronom personnel renvoie toujours à une personne bien déterminée sur le plan du genre et du nombre.

C'est là peut-être une référence à la grammaire arabe où l'identité de tout pronom personnel est contenue dans sa forme grammaticale et dans sa conjugaison. Cette lacune a eu une répercussion sur la suite de la leçon; elle a mis le groupe-classe en difficulté lors de la transformation passive de la $3^{3}$ phrase du corpus, comme cela ressort nettement de l'extrait de conversation suivant :

$\mathrm{P}$ : Qui veut transformer la troisième phrase?

E : L'équipe de flage a été battu par On.

$P$ : Nous avons dit que «On » est un pronom indéfini qui ne renvoie pas à un agent bien déterminé.

Est-ce nous savons qui a battu l'équipe de flage?

E : Non.

P: Dans ce cas, nous n'exprimerons pas l'agent de l'action, il s'agit d'un cas d'effacement du complément d'agent. Retenons donc que chaque fois qu'on a une phrase active qui a pour sujet on, la phrase passive qui lui correspond est marquée par l'absence du complément d'agent.

La maîtrise d'une opération comme la transformation passive présuppose la présence de certaines connaissances de base. En conséquence, la moindre lacune dans les connaissances grammaticales des élèves entraîne un gaspillage de temps, l'enseignant étant obligé d'ouvrir des parenthèses pour expliquer des points grammaticaux périphériques par rapport à l'objectif de la leçon.

\subsubsection{Amalgame des types et des formes}

Les termes «types de phrase " et «formes de phrase » font partie de la terminologie de la grammaire scolaire. Les types et les formes sont organisés de façon différente: les premiers sont "exclusifs", c'est-à-dire qu'une même phrase correspond à un seul type, les secondes, au contraire, se combinent entre elles, c'est-à-dire que, dans un même énoncé, on peut assister à la réalisation de plusieurs formes. En outre, les formes de phrase se construisent à partir des types par transformations formelles, morphologiques ou syntaxiques.

Ces connaissances doivent être maîtrisées par les élèves de la classe terminale du collège, ce qui n'est pas le cas d'après les échanges qui suivent :

$P$ : Qu'est-ce qu'on appelle une phrase dont le sujet fait l'action?

Quelle est la forme de ces phrases?

$\mathrm{E}:$ Ce sont des phrases déclaratives.

$\mathrm{P}$ : Oui, ce sont des phrases déclaratives, mais nous cherchons leur forme.

Quelles sont les formes de phrase que vous connaissez? 
E : Phrase affirmative et phrase négative.

$P$ : Il y a aussi la phrase active et la phrase passive. Dans le cas où le sujet fait

l'action, il s'agit d'une phrase active et dans le cas où le sujet subit l'action, il s'agit d'une phrase passive.

S'agit-il ici de phrase active ou passive?

E : Lorsque le sujet fait l'action, il s'agit d'une phrase active.

La réponse de l'élève qui a pris la parole au début témoigne de la confusion des types et des formes dans l'esprit de tous les élèves de la classe, aucun d'entre eux ne l'ayant corrigé. Ce n'est qu'une fois l'explication fournie par le professeur qu'un autre élève donne une réponse significative quant à la connaissance du groupeclasse au sujet des formes de phrase. II apparaît que les élèves ne connaissent que la forme affirmative et la forme négative. C'est logique, car ils n'ont vu, au cours des trois années du collège, que ces deux formes.

Dans les programmes officiels, qui font autorité parmi les enseignants, on prévoit une leçon sous l'intitulé "les types et les formes de phrase », où sont abordés les quatre types mais où on n'enseigne que les deux formes dont on a parlé. En 8 année, on envisage une leçon sous le titre "la forme affirmative et la forme négative $\%$.

Ce qui est incompréhensible à cet égard, c'est qu'on programme en qe année une leçon sur la transformation passive alors qu'on n'a pas initié les élèves à cette forme de phrase au cours de la 7 e ou de la 8e année. La seule explication plausible à cette situation est que les concepteurs des programmes de chaque niveau d'enseignement travaillent indépendamment les uns des autres.

\subsubsection{Difficultés dans la reconnaissance de certaines fonctions grammaticales}

Après avoir expliqué le processus de transformation passive et avant de l'appliquer aux phrases du corpus, l'enseignant procède à une analyse en constituants immédiats qui fait apparaître des lacunes dans l'identification de certaines fonctions grammaticales, comme le montrent les exemples suivants :

$P$ : Où est le G.N.S dans la phrase 4 ?

E : Robert.

$P$ : Où est le groupe verbal?

$\mathrm{E}$ : Parle à sa mère.

$P$ : ll est constitué de quoi ?

E : Un verbe.

$P$ : Quel est ce verbe?

E : Parle.

$P$ : Quel est l'autre élément qui forme ce groupe verbal?

E : C.O.D.

$P$ : Où est-il ce C.O.D ? 
$E$ : Sa mère.

$P$ : Regardez bien. Qu'est-ce qu'il y a entre le verbe « parle » et « sa mère »?

$E: \grave{A}$.

$\mathrm{P}:$ «À est une préposition qui sépare le verbe du groupe nominal "sa mère ॥; celui-ci forme avec la préposition « à » un complément d'objet indirect.

Nous pensons que cette difficulté à reconnaître le C.O.D et que l'incapacité à le différencier du C.O.I s'expliquent par le fait qu'on n'a pas beaucoup insisté sur l'analyse en constituants immédiats au collège, mais surtout par la négligence d'une question très importante dans la grammaire de la phrase : celle de la transitivité des verbes, dont la maîtrise facilite la reconnaissance des compléments essentiels. L'autre fonction grammaticale qui a échappé à la connaissance des élèves est celle du complément d'agent.

$P$ : Que devient le sujet de la phrase active?

$E$ : Absence de réponse.

$\mathrm{P}:$ Le sujet de la phrase active devient complément d'agent de la phrase passive. Il est généralement introduit par la préposition par.

L'absence de réponse est justifiée par le fait qu'il s'agit d'une nouvelle notion grammaticale pour les élèves, ne faisant pas partie de leur «patrimoine métalinguistique $\%$.

II convient de remarquer, à la fin de cet inventaire des défaillances grammaticales des collégiens, que celles-ci sont dues à la nouvelle répartition des contenus qui ne respecte pas l'ordre d'acquisition des formes linguistiques.

\subsection{Techniques d'enseignement et discours grammatical de l'enseignant}

En situation de classe, l'enseignant est contraint de chercher à concilier les besoins de l'apprenant et le contenu d'enseignement, c'est-à-dire qu'il se doit de trouver les moyens pédagogiques efficaces pour subvenir aux besoins de l'acte d'enseignement finalisé et orienté vers un ou plusieurs objectifs.

La recherche en didactique des langues a pu définir le statut de l'apprenant en le mettant au centre du processus d'enseignement-apprentissage, mais elle n'a pas pour autant suffisamment exploré le rôle de l'enseignant. Or le comportement pédagogique de l'enseignant constitue le garant de la réussite de la leçon et d'une transmission fiable du savoir. Parmi les composantes qui constituent l'activité enseignante, ce sont les techniques d'enseignement qui semblent les plus importantes car elles dessinent le contour de la situation d'apprentissage.

Néanmoins, la littérature didactique manque d'études qui interrogent les pratiques grammaticales en classe de L2. Malgré ce manque, la recherche empirique entreprise par Peck (1988) pour détecter les techniques d'enseignement de la grammaire en classe de $L_{2}$ s'est révélée brillante par les résultats auxquels ce didacticien a abouti.

Les observations de classes effectuées par ce chercheur lui ont permis de dégager cinq types majeurs de techniques d'enseignement de la 
grammaire: identification, classification, systématisation, application, et généralisation.

Bien entendu, ces techniques ne se retrouvent pas tous chez un même enseignant, c'est-à-dire que le recours à tel ou tel procédé varie selon les cas. Si on examine le cas particulier de l'enseignant qu'on a observé, on se rend compte qu'il fait tout d'abord identifier un certain nombre de phrases actives destinées à être transformées à la forme passive. Il procède ensuite à une systématisation : comme toutes les phrases contenant un C.O.D. admettent la transformation passive, il explique comment on passe de la forme active à la forme passive(les modifications syntaxiques qui assurent le passage à la forme passive). L'enseignant prévoit ensuite une phase d'application visant à fixer l'acquis principal de la leçon en proposant deux exercices figurant dans le manuel, qui invitent les élèves à transformer des phrases actives à la forme passive.

La dernière étape consiste à formuler oralement un paragraphe résumant le processus de la transformation passive, qui sera par la suite inscrit au tableau et recopié par les élèves sous forme de synthèse écrite; il y a là une sorte de généralisation. En tout, notre enseignant a donc eu recours à quatre techniques différentes de présentation:identification, systématisation, application et généralisation, parmi lesquelles l'identification a été utilisée à plusieurs reprises.

En dépit des techniques d'enseignement, l'enseignant façonne à sa manière le savoir grammatical et transforme le contenu des grammaires et des manuels pour s'ajuster au niveau des élèves et à leurs connaissances antérieures. L'ensemble de ces transformations constitue le discours grammatical de l'enseignant qui comporte un certain nombre de caractéristiques. Certaines de ces caractéristiques apparaissent dans le discours de l'enseignant que nous avons observé. La caractéristique la plus redondante est illustrée par l'exemple suivant :

P : Que désigne On? Une personne ou plusieurs personnes?

E : Une personne.

$\mathrm{P}$ : Comment vous le savez?

E : Il se conjugue au singulier.

$P$ : Est-ce qu'on connaît précisément cette personne? Est-ce qu'on peut remplacer « on » par un groupe nominal ?

E : Non.

$P$ : Donc il s'agit d'un pronom personnel inconnu, nous ne pouvons pas l'identifier, il peut désigner une ou plusieurs personnes, c'est un pronom personnel indéfini.

L'enseignant procède à une explication en opérant un tri dans ses connaissances pour fournir aux apprenants l'information qui lui paraît la plus adéquate à leur «patrimoine métalinguistique». "I a remarqué, en effet, que les élèves ne connaissent pas le terme "pronom personnel indéfini», et il a utilisé le vocable courant « inconnu » pour se rapprocher de l'état de leurs connaissances. Ce recours à la simplification grammaticale traduit un choix pédagogique de l'enseignant ainsi qu'une tendance à prendre en compte « le bagage grammatical » des élèves et leur niveau de connaissances grammaticales dans leur $L_{1}$. 
Comme nous l'avons déjà mentionné, la grammaire arabe ne contient pas ce genre de pronom où l'on occulte l'agent de l'action.

D'ailleurs, le terme « inconnu » employé par l'enseignant pour désigner on est une traduction de la lexie désignant la seule forme grammaticale arabe où l'on n'exprime pas l'agent de l'action (mabni lil mažhul).

L'on peut voir une autre manifestation du discours grammatical de l'enseignant à travers l'extrait suivant:

$P$ : Qui transforme la phrase?

$\mathrm{E}$ : L'exercice a été fait par les élèves.

$P$ : Attention au temps.

E : L'exercice fera par les élèves.

$P$ : L'exercice sera fait par les élèves.

Dans cet exemple, l'enseignant demande aux élèves de transformer la $2^{e}$ phrase du corpus à la forme passive. Nous remarquons que la réponse escomptée est souvent donnée par le professeur à la suite d'un ou de deux essais infructueux. Au lieu de rappeler la règle grammaticale qui régit la transformation du temps, l'enseignant donne une phrase type.

Il fait usage également d'un style télégraphique qui n'a rien à voir avec les grammaires élaborées par les spécialistes. En résumé, le langage de l'enseignant est jalonné de conventions, d'expressions qui se substituent aux règles. Ce langage est qualifié de «paragrammatical » car il est constitué de comparaisons, de métaphores et de simplifications. C'est dans ce sens que langage de l'enseignant s'éloigne des descriptions grammaticales du linguiste ou du grammairien.

Il est vrai en revanche, que le recours au métalangage des linguistes, sans aucune modification, risque de compliquer les choses et de dérouter les élèves; d'un autre côté cependant, le langage "paragrammatical " de l'enseignant renforce la tendance de l'apprenant à puiser dans le métalangage de sa langue maternelle et réduit l'efficacité des explications grammaticales.

La troisième caractéristique du métalangage de l'enseignant consiste à commenter une forme grammaticale, comme c'est le cas dans l'exemple suivant :

$P$ : La forme verbale "sera fait » est une construction passive. C'est une forme grammaticale costruite à partir de la transformation du temps du verbe "faire » lors du passage de la forme active à la forme passive.

Vous devez normalement reconnaître cette structure ainsi que le comment de sa formation.

La transformation passive d'une phrase active dont le verbe est mis au futur simple de l'indicatif nous appelle à conjuguer ce verbe au temps composé qui lui correspond.

La défaillance dans la reconnaissance de cette structure a poussé l'enseignant à s'attarder sur ce point grammatical pour mieux expliquer le phénomène linguistique en question sous la forme d'un commentaire d'ordre pédagogique. 
Outre ces trois caractéristiques, l'enseignant de L2 peut aussi préparer des situations-exemples ou recourir à des explications grammaticales reposant sur l'éclectisme, comme l'ont montré les quelques rares études empiriques sur la question. Les remarques et les analyses qui précèdent montrent que ce qu'on appelle "la grammaire interactive" et qui a lieu en salle de classe, diffère significativement des écrits des grammairiens et des didacticiens. Pour appuyer cette réalité, nous rapportons la synthèse écrite formulée par le groupe-classe à la fin de la séance tout en procédant à une comparaison avec le savoir proposé par le manuel :

Pour transformer une phrase de la forme active à la forme passive :

- Le C.O.D de la phrase active devient le sujet de la phrase passive.

- Le verbe se transforme : il est formé de l'auxiliaire "être " conjugué au même temps que le verbe de la phrase active + le participe passé du verbe.

- Le sujet de la phrase active devient le complément d'agent de la phrase passive.

- Seules les phrases qui contiennent un C.O.D peuvent être transformées à la forme passive.

- Quand le sujet de la phrase est le pronom indéfini on, on doit effacer le complément d'agent.

Par rapport au manuel, cette synthèse présente deux différences principales:

- Tandis que l'enseignant se limite à un seul cas d'effacement du complément d'agent (le cas du pronom personnel indéfini on), le manuel énumère quatre constructions illustrant ce phénomène.

- Alors que le manuel cite trois cas où il est impossible d'opérer la transformation passive (le verbe de la phrase active est transitif indirect, ou pronominal, le cas où le verbe de la phrase active n'a pas le même sens que lorsqu'il est employé dans une phrase passive), l'enseignant n'envisage qu'un seul cas.

- Il convient de remarquer aussi que l'enseignant fait référence à un savoir traditionnel lorsqu'il définit la phrase active comme étant « la phrase dont le sujet fait l'action » ou la phrase passive comme étant "la phrase dont le sujet subit l'action " . C'est une définition qui ne figure ni dans le manuel actuel ni dans les grammaires de référence à base linguistique.

C'est là l'influence de sa formation académique et de la conception qu'il se fait de la grammaire. Dans le même ordre d'idée, nous avons constaté que l'enseignant réalise la phase d'application ou d'exercisation à la fin de la séance, c'est-à-dire après l'achèvement de l'étape d'explication, ce qui signifie qu'il adopte la conception béhavioriste de l'apprentissage, contrairement au manuel qui met l'exercice au service de l'extraction des règles, conformément au point de vue constructiviste. Enfin, nous pouvons dire que tout comme le manuel, l'enseignant n'a fait aucune allusion à la relation que peut entretenir la phrase passive avec l'architecture textuelle. 


\section{Conclusion}

Aborder la grammaire dans sa structuration interne, indépendamment de l'implication de l'apprenant dans la construction du savoir, c'est, finalement, considérer ce dernier comme un simple receveur de connaissances. Or, les nouvelles orientations didactiques mettent l'apprenant au centre de l'opération d'enseignement-apprentissage, ce qui exige de l'enseignant qu'il cède l'initiative à ses élèves pour qu'ils construisent eux-mêmes leurs propres connaissances.

La grammaire enseignée effectivement en classe, avons-nous dit, participe à la construction de l'interlangue des apprenants. En effet, l'observation d'une séance de grammaire révèle quelques défaillances dans les connaissances grammaticales des élèves dont certaines sont dues à l'organisation des contenus d'enseignement de cette matière. C'est ainsi qu'une restructuration de l'enseignement grammatical s'avère nécessaire pour régler certains problèmes marquant l'itinéraire d'apprentissage du système linguistique de la langue cible. Si les enseignants disposent aujourd'hui d'une variété de méthodes d'enseignement de la grammaire, ils doivent opter pour les meilleurs choix afin d'optimiser les apprentissages linguistiques en classe de FLE.

\section{Bibliographie}

BEACCO, J.-C. (1995). La méthode circulante et les méthodologies constituées. Le français dans le monde, numéro spécial, pp. 42-49.

Cicurel, F. (1985). Parole sur parole. Le métalangage en classe de langue. Paris : CLE international.

COURTILLON, J. (1989). La grammaire sémantique et l'approche communicative. Le français dans le monde, numéro spécial, pp. 113-122.

CUQ, J.-P. (1996). Une introduction à la didactique de la grammaire en français langue étrangère. Paris : Didier-Hatier.

GERMAIN, C. \& SEGUIN, H. (1998). Le point sur la grammaire. Paris : CLE International.

PECK, A. (1998). Language teachers at work: description of methods. New York : Prentice Hall.

Riegel, M., Pellat, J.-C., Rioul, R. (1994). La grammaire méthodique du français. Paris : PUF.

ROULEt, E. (1972). Théories grammaticales, descriptions et enseignement des langues. Paris : Nathan.

VIGNER, G. (1984). L'exercice en classe de français. Paris: Hachette.

- (2004). La grammaire en FLE. Paris : Hachette. 